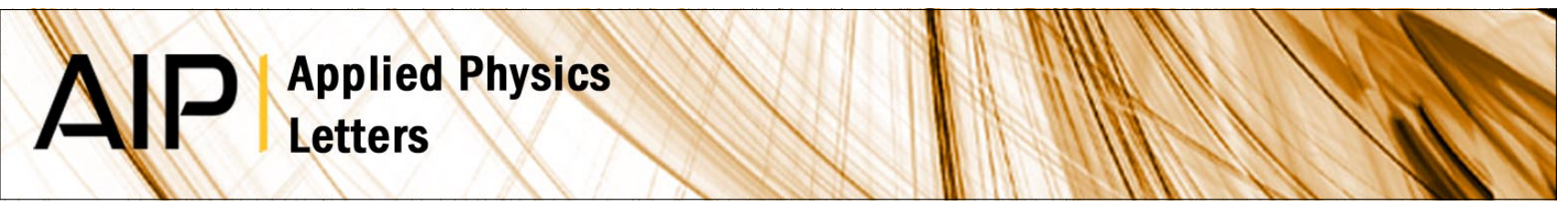

\title{
Self-assembled oxide nanopillars in epitaxial BaFe2As2 thin films for vortex pinning
}

Yi Zhang, Christopher T. Nelson, Sanghan Lee, Jianyi Jiang, Chung Wung Bark et al.

Citation: Appl. Phys. Lett. 98, 042509 (2011); doi: 10.1063/1.3532107

View online: http://dx.doi.org/10.1063/1.3532107

View Table of Contents: http://apl.aip.org/resource/1/APPLAB/v98/i4

Published by the AIP Publishing LLC.

Additional information on Appl. Phys. Lett.

Journal Homepage: http://apl.aip.org/

Journal Information: http://apl.aip.org/about/about_the_journal

Top downloads: http://apl.aip.org/features/most_downloaded

Information for Authors: http://apl.aip.org/authors

\section{ADVERTISEMENT}
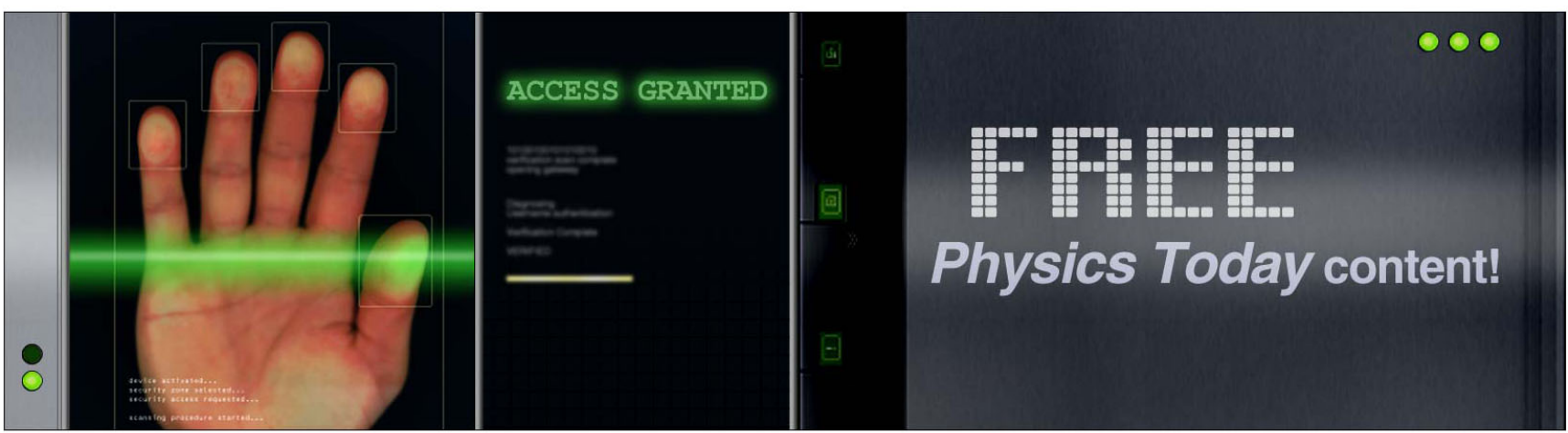


\title{
Self-assembled oxide nanopillars in epitaxial $\mathrm{BaFe}_{2} \mathrm{As}_{2}$ thin films for vortex pinning
}

\author{
Yi Zhang, ${ }^{1,2}$ Christopher T. Nelson, ${ }^{2}$ Sanghan Lee, ${ }^{3}$ Jianyi Jiang, ${ }^{4}$ Chung Wung Bark, ${ }^{3}$ \\ Jeremy D. Weiss, ${ }^{4}$ Chiara Tarantini, ${ }^{4}$ Chad M. Folkman, ${ }^{3}$ Seung-Hyub Baek, ${ }^{3}$ \\ Eric E. Hellstrom, ${ }^{4}$ David C. Larbalestier, ${ }^{4}$ Chang-Beom Eom, ${ }^{3}$ and Xiaoqing Pan $^{2, a)}$ \\ ${ }_{1}^{1}$ Department of Materials Science and Engineering and National Laboratory of Solid State Microstructures, \\ Nanjing University, Nanjing 210093, People's Republic of China \\ ${ }^{2}$ Department of Materials Science and Engineering, University of Michigan, Ann Arbor, \\ Michigan 48109, USA \\ ${ }^{3}$ Department of Materials Science and Engineering, University of Wisconsin-Madison, Madison, \\ Wisconsin 53706, USA \\ ${ }^{4}$ Applied Superconductivity Center, National High Magnetic Field Laboratory, Florida State University, \\ Tallahassee, Florida 32310, USA
}

(Received 1 November 2010; accepted 1 December 2010; published online 27 January 2011)

\begin{abstract}
We report the structure and chemistry of the self-assembled oxide nanopillars that form in superconducting Co-doped $\mathrm{BaFe}_{2} \mathrm{As}_{2}$ thin film grown by pulsed laser deposition. The oxide nanopillars consist of a $\mathrm{BaFeO}_{2}$ phase, form epitaxially on the $\mathrm{SrTiO}_{3}$ template, and grow coherently with the $\mathrm{BaFe}_{2} \mathrm{As}_{2}$ film. The nanopillars are square with a uniform size of 4-5 nm, which is close to twice the superconducting coherence length. Despite a volume content of $\sim 5 \%$, the nanopillars do not degrade the structural quality of the $\mathrm{BaFe}_{2} \mathrm{As}_{2}$ matrix. Indeed the nanopillars provide exceptionally strong vortex pinning and high critical current density due to the very close correlation of pillar and vortex core diameters. (c) 2011 American Institute of Physics.

[doi:10.1063/1.3532107]
\end{abstract}

In the past years major efforts have been invested in optimizing high-temperature superconductors (HTSs) by increasing their critical current density $J_{c}$ and reducing their anisotropy. These goals require the introduction of nanostructures able to strongly pin magnetic flux vortices so as to oppose vortex motion induced by the Lorentz force. This approach has been applied in cuprate HTS. For instance, several works on $\mathrm{YBa}_{2} \mathrm{Cu}_{3} \mathrm{O}_{7}$ (YBCO) report a substantial improvement of pinning properties incorporating nonsuperconducting rare-earth oxide $\left(\mathrm{RE}_{2} \mathrm{O}_{3}\right)$ precipitates and $\mathrm{BaZrO}_{3}$ nanopillars, ${ }^{1}$ but often with a critical temperature suppression when the $\mathrm{BaZrO}_{3}$ content exceeds $1 \%-2 \%$. In general, optimum vortex pinning is achieved by a high density of defects whose size is comparable to twice the superconducting coherence length $\xi$, especially when elongated along the applied magnetic field direction. ${ }^{2}$

The pinning mechanisms have not yet been fully understood in the recently discovered iron-based superconductors, promising for their high critical temperature $T_{c}$, low anisotropy, extremely high upper critical magnetic field $H_{c 2}$, and irreversibility field $H_{\text {irr. }}{ }^{3-11}$ Recently, we have observed correlated line defects in thin films of the Co-doped superconductor $\mathrm{BaFe}_{2} \mathrm{As}_{2}(\mathrm{Ba}-122)$ grown on $\mathrm{SrTiO}_{3}$ (STO) surfaces which exhibit strong and anisotropic vortex pinning. ${ }^{12}$ In this work we report on the structure, composition, and growth mechanisms of these nanopillars using subangstrom resolution transmission electron microscopy (TEM) and electron energy-loss spectroscopy (EELS). The TEM shows how a high density of nanopillars can be incorporated in the Ba-122 films while producing very strong vortex pinning and very high $J_{c}$ without degrading the structural properties of the superconducting matrix.

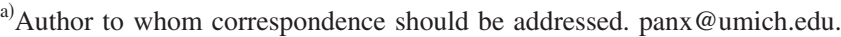

The Co-doped Ba-122 thin film with a thickness of 350 nm was grown on 100 unit cell (u.c.) STO templated (001) $(\mathrm{La}, \mathrm{Sr})(\mathrm{Al}, \mathrm{Ta}) \mathrm{O}_{3}(\mathrm{LSAT})$ substrates in vacuum at $750{ }^{\circ} \mathrm{C}$ by pulsed laser deposition (PLD). The detailed growth conditions are reported elsewhere. ${ }^{13,14}$ TEM studies were obtained with JEOL 3011 and an aberration-corrected JEOL ARM-200F electron microscopes.

The microstructure of the Co-doped Ba-122 films grown on 100 u.c. STO/LSAT is shown in the cross-sectional TEM image of Fig. 1(a) viewed along the [100] zone axis. The corresponding selected-area electron diffraction (SAED) pattern inset at the top left shows that the Ba-122 film is single crystal and epitaxial to the STO/LSAT substrate. A moderate density of vertical line defects running along the $c$-axis of the Ba-122 film can be seen in the TEM image. A magnified region of the line defects near the film/STO interface shown in Fig. 1(b) reveals that these nanosized pillars nucleate at the Ba-122/STO interface. The uniformly distributed nanopillars with an average spacing of $\sim 10 \mathrm{~nm}$ can be clearly seen in the planar view scanning transmission electron microscopy (STEM) image in Fig. 1(c). The high resolution STEM image in Fig. 1(d) shows that nanopillars are square with a uniform size of $4-5 \mathrm{~nm}$ and are also epitaxial with respect both to the surrounding Ba-122 matrix and the STO template. Figure 1(e) shows the high angle annular dark field (HAADF) image of a nanopillar in the Ba-122 film, in which the image contrast is determined by the atomic number of element (Z-contrast image). It is seen that the nanopillar has an ordered crystalline structure which is different from the Ba-122 structure.

The chemical composition of nanopillars was determined using EELS. The region analyzed is shown in the Z-contrast image [Fig. 2(a)] which includes both a nanopillar and Ba-122 matrix. Figure 2(b) shows the variation of the atomic ratio of $\mathrm{Ba}, \mathrm{Fe}, \mathrm{As}$, and $\mathrm{O}$ atoms along the horizontal 

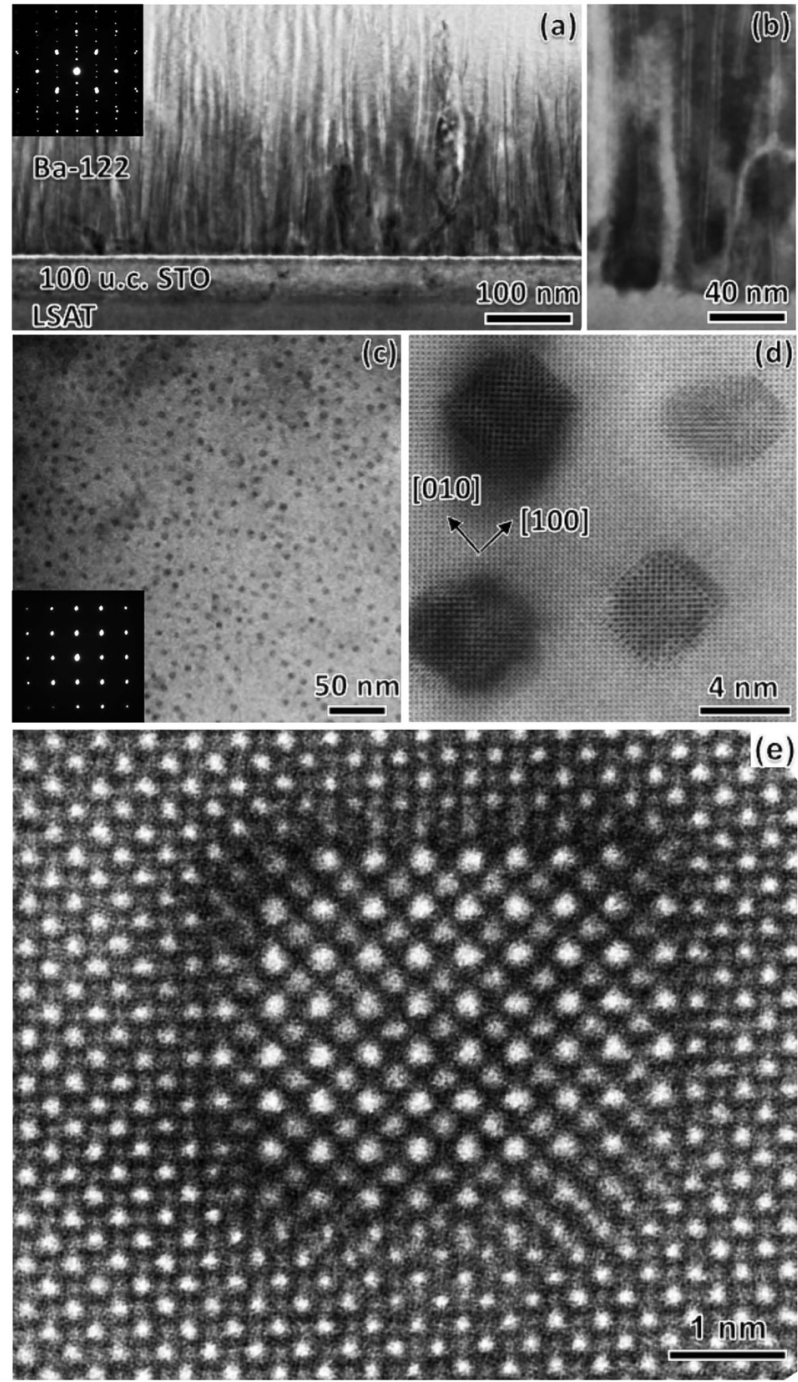

FIG. 1. (a) The cross-sectional TEM image of Co-doped Ba-122 film grown on 100 u.c. STO/LSAT. This image is viewed along the [100] zone axis. The inset at the top left is the corresponding SAED pattern. (b) A magnified region of the line defects near the film/STO interface. (c) The planar view bright field TEM image viewed along the [001] zone axis. The corresponding SAED pattern inset at the bottom left shows a single crystal pattern. (d) A high resolution STEM image of (c) showing the structure of the nanopillars in the Ba-122 film. (e) A high angle annular dark field (Z-contrast) image showing the atomic structure of an epitaxial nanopillar in the Ba-122 film.
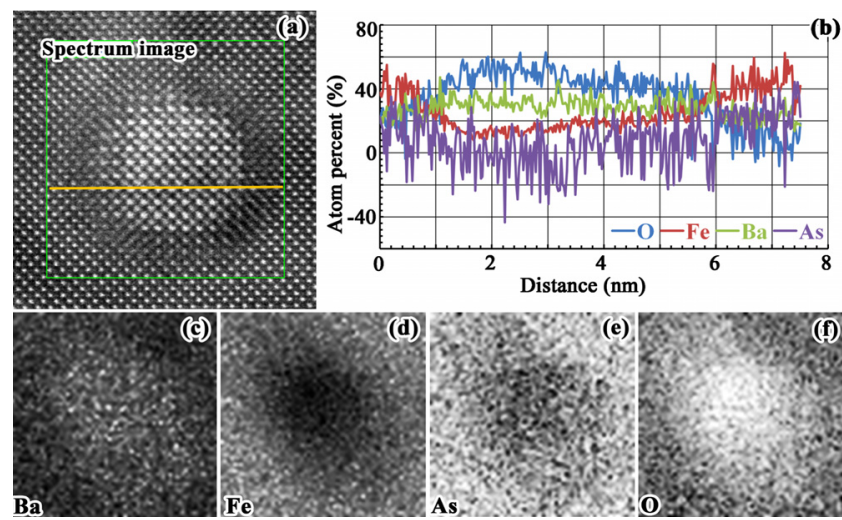

FIG. 2. (Color online) (a) HAADF image showing the analyzed region which includes both a nanopillar and the Ba-122 matrix. (b) The change of the atomic ratio of $\mathrm{Ba}, \mathrm{Fe}, \mathrm{As}$, and $\mathrm{O}$ along the line in (a). Relative composition maps for $\mathrm{Ba}, \mathrm{Fe}, \mathrm{As}$, and $\mathrm{O}$ are shown in (c)-(f), respectively.

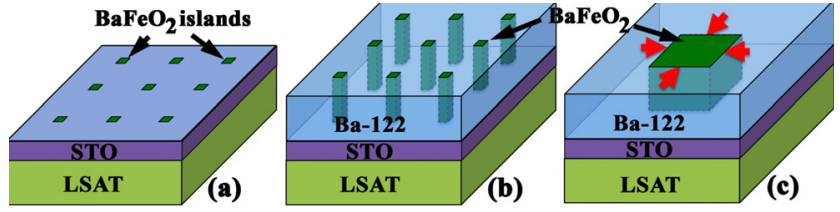

FIG. 3. (Color online) Schematic models showing the growth process of the $\mathrm{BaFeO}_{2}$ nanopillars in the $\mathrm{Ba}-122$ matrix. (a) Nanosized oxide islands nucleate on the surface of STO template. (b) Nanopillars of uniform diameter embed in the fully grown Ba-122 matrix. (c) The nanopillar is coherently strained by the surrounding $\mathrm{Ba}-122$ matrix.

line in Fig. 2(a). Both the line scan [Fig. 2(b)] and twodimensional maps [Figs. 2(c)-2(f)] show that the nanopillar is a $\mathrm{Ba}-\mathrm{Fe}-\mathrm{O}$ phase that does not contain As. Quantitative analysis suggests that the composition ratio of $\mathrm{Ba}: \mathrm{Fe}: \mathrm{O}$ varies from 1:1:2 to 1.4: 1: 2.8. Searching $\mathrm{Ba}-\mathrm{Fe}-\mathrm{O}$ compounds we found $\mathrm{BaFeO}_{3}$, a pseudocubic perovskite, with a lattice parameter $a=4.210 \AA$, which was reported to form on the (001) STO substrate by PLD. ${ }^{15}$ The x-ray diffraction patterns of our films typically show a metallic Fe peak, ${ }^{14}$ whereas $\mathrm{Fe}$ in $\mathrm{BaFeO}_{3}$ has an oxidation state of $\mathrm{Fe}^{4+}$. The $\mathrm{Fe}-\mathrm{O}$ phase diagram ${ }^{16}$ shows that metallic $\mathrm{Fe}$ cannot coexist with $\mathrm{Fe}^{4+}$. Thus based on thermodynamic arguments, the nanopillars cannot be $\mathrm{BaFeO}_{3}$.

We expanded our search to $\mathrm{Sr}-\mathrm{Fe}-\mathrm{O}$ compounds to see if there are $\mathrm{Sr}$-analogs of not-yet-reported $\mathrm{Ba}-\mathrm{Fe}-\mathrm{O}$ compounds. We found $\mathrm{SrFeO}_{2}$ (Ref. 17) and $\mathrm{Sr}_{3} \mathrm{Fe}_{2} \mathrm{O}_{5},{ }^{18}$ which both contain $\mathrm{Fe}^{2+} . \mathrm{Sr}_{3} \mathrm{Fe}_{2} \mathrm{O}_{5}$ is an orthorhombic doublelayered perovskite $(a=3.5148 \AA, b=3.9527 \AA$, and $c$ $=20.9125 \AA$ ) with the Immm space group (No. 71), which does not match the square shape of nanopillars observed by TEM. On the other hand, $\mathrm{SrFeO}_{2}$ has a tetragonal structure $(a=3.991 \AA, c=3.474 \AA$ ) with the $P 4 / \mathrm{mmm}$ space group. The simulated Z-contrast images of $\mathrm{SrFeO}_{2}$ match images of the nanopillars. We believe that the nanopillars are nonstoichiometric $\mathrm{BaFeO}_{2}$, which has not yet been reported in the literature.

The formation of self-assembled, vertically aligned epitaxial $\mathrm{BaFeO}_{2}$ nanopillars in the $\mathrm{Ba}-122$ matrix can be attributed to the structurally similar perovskite surface of STO and the residual oxygen incorporated in PLD target. The intrinsic similarities in the structure and chemistry of the $\mathrm{BaFeO}_{2}$ nanopillars favor nucleation of nanosized oxide islands on the perovskite STO template, as shown schematically in Fig. 3(a). The surface energy plays the primary role in controlling the self-assembled nanopillars. ${ }^{19}$ Bulk $\mathrm{BaFe}_{2} \mathrm{As}_{2}$ has a tetragonal structure $(I 4 / \mathrm{mmm})$ with lattice parameters $a$ $=3.962 \AA$ and $c=13.017 \AA{ }^{11}$ and $\mathrm{BaFeO}_{2}$ has a tetragonal structure with lattice parameters $a>3.991 \AA$ and $c$ $>3.474 \AA$ according to that of $\mathrm{SrFeO}_{2}$ with the substitution of Sr by larger Ba. The bulk STO has a lattice constant with $a=3.905 \AA$, but the thin STO templates are coherently strained to match the lattice constant of LSAT $(a=3.869 \AA) .{ }^{14}$ The misfit between $\mathrm{BaFe}_{2} \mathrm{As}_{2}$ and the LSAT substrate is only $2.3 \%$, whereas the misfit between $\mathrm{BaFeO}_{2}$ and LSAT is $>3.2 \%$. Thus, the nanopillars nucleate and grow under compression from both the substrate and the surrounding Ba-122 matrix, as schematically shown in Fig. 3(c).

In Ref. 12 we showed a strong vortex pinning along the $c$-axis which requires the presence of vortex pinning centers whose size is about $2 \xi$, where the coherence length $\xi$ is $2.5-3$ $\mathrm{nm}$ for Ba-122. Thus, the microscopy shows that the typical 


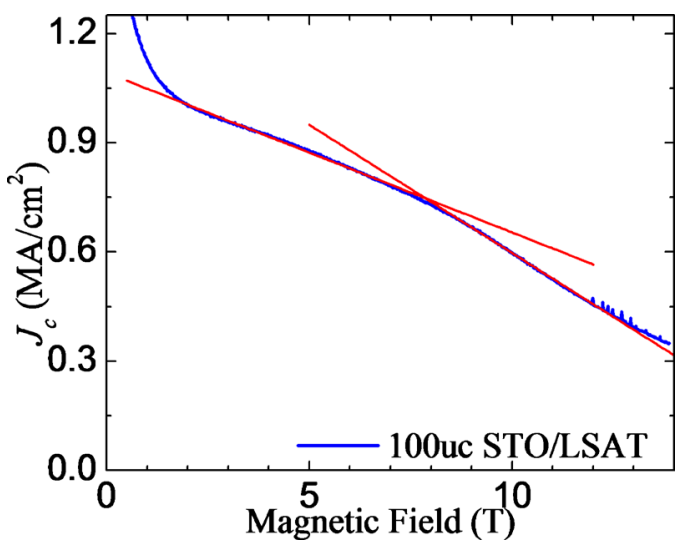

FIG. 4. (Color online) Magnetization $J_{c}$ as a function of magnetic field at $4.2 \mathrm{~K}$ with the field applied perpendicular to the plane of the film for the Ba-122 film on 100 u.c. STO/LSAT. The change in slope at $\sim 8.5 \mathrm{~T}$ corresponds to $H_{M}$.

oxide nanopillars give rise to almost perfect pinning centers for this material. Judged by the lack of $T_{c}$ suppression in these films and the general properties of oxides, we anticipate that the nanopillars are insulating and thus provide a strong binding force to the vortex cores. From Fig. 1(c) we estimate a density of nanopillars of $\Phi=4.1 \times 10^{-3} \mathrm{~nm}^{-2}$. Assuming that the strongest pinning corresponds to one vortex residing on each nanopillar, this value of $\Phi$ corresponds to a matching field of $\mu_{0} H_{M}=8.5 \mathrm{~T}$. Figure 4 shows that $J_{c}$ as a function of $H$ clearly changes slope at the same field $\sim 8.5 \mathrm{~T}$. Up to $H_{M}$ all vortices are located in the nanopillars and are strongly pinned, meaning that $J_{c}$ is only slightly suppressed by increasing field. However, above $H_{M}$ vortices must reside between the nanopillars where only weak pinning centers and vortex-vortex interactions prevent their motion. Such vortices are more easily depinned and $J_{c}$ drops more rapidly.

In conclusion, we provided a detailed TEM analysis of the oxide nanopillars that form in $\mathrm{Ba}-122$ films grown on STO templated LSAT substrates when small oxygen content is present. The oxide nanopillars form an epitaxial $\mathrm{BaFeO}_{2}$ phase that nucleates on the surface of the STO template since they have the similar structure and chemistry at their interface. We propose that the in-plane compressive strain caused by the misfit between $\mathrm{BaFeO}_{2}$ and the substrate restricts the lateral size of the nanopillars to around 10 u.c. and drives them to grow along the $c$-axis forming a nanopillar structure. The high density of self-assembled nonsuperconducting oxide nanopillars provides very effective flux pinning centers in the Ba-122. The use of self-assembled nanopillars as correlated defects for strong vortex pinning provides an efficient method for increasing $J_{c}$ without any apparent film thickness dependence or need for postprocessing. Different from YBCO with $\mathrm{BaZrO}_{3}$, where the nanopillars generally de- grade the structural uniformity of YBCO matrix and suppress $T_{c}$ by a few degrees, in Ba-122 thin films the matrix maintains its properties with the addition of strong pinning centers in spite of the high concentration of nanopillars.

This work was supported by the Department of Energy under Grant Nos. DE-FG02-07ER46416 and DE-FG0206ER46327, NSF under Award Nos. DMR-0907191, DMR0723032, DMR-0820404, and DMR-0084173, and AFOSR under Grant No. FA9550-06-1-0474.

${ }^{1}$ S. R. Foltyn, L. Civale, J. L. Macmanus-Driscoll, Q. X. Jia, B. Maiorov, H. Wang, and M. Maley, Nature Mater. 6, 631 (2007).

${ }^{2}$ L. Civale, A. D. Marwick, T. K. Worthington, M. A. Kirk, J. R. Thompson, L. Krusin-Elbaum, Y. Sun, J. R. Clem, and F. Holtzberg, Phys. Rev. Lett. 67, 648 (1991).

${ }^{3}$ Y. Kamihara, T. Watanabe, M. Hirano, and H. Hosono, J. Am. Chem. Soc. 130, 3296 (2008)

${ }^{4}$ X. H. Chen, T. Wu, G. Wu, R. H. Liu, H. Chen, and D. F. Fang, Nature (London) 453, 761 (2008).

${ }^{5}$ C. Wang, L. J. Li, S. Chi, Z. W. Zhu, Z. Ren, Y. K. Li, Y. T. Wang, X. Lin, Y. K. Luo, S. Jiang, X. F. Xu, G. H. Cao, and Z. A. Xu, EPL 83, 67006 (2008).

${ }^{6}$ M. Rotter, M. Tegel, and D. Johrendt, Phys. Rev. Lett. 101, 107006 (2008).

${ }^{7}$ K. Iida, J. Hanisch, R. Huhne, F. Kurth, M. Kidszun, S. Haindl, J. Werner, L. Schultz, and B. Holzapfel, Appl. Phys. Lett. 95, 192501 (2009).

${ }^{8}$ H. Q. Yuan, J. Singleton, F. F. Balakirev, S. A. Baily, G. F. Chen, J. L. Luo, and N. L. Wang, Nature (London) 457, 565 (2009).

${ }^{9}$ F. Hunte, J. Jaroszynski, A. Gurevich, D. C. Larbalestier, R. Jin, A. S. Sefat, M. A. McGuire, B. C. Sales, D. K. Christen, and D. Mandrus, Nature (London) 453, 903 (2008).

${ }^{10}$ A. Yamamoto, J. Jaroszynski, C. Tarantini, L. Balicas, J. Jiang, A. Gurevich, D. C. Larbalestier, R. Jin, A. S. Sefat, M. A. McGuire, B. C. Sales, D. K. Christen, and D. Mandrus, Appl. Phys. Lett. 94, 062511 (2009).

${ }^{11}$ M. Rotter, M. Tegel, D. Johrendt, I. Schellenberg, W. Hermes, and R. Pottgen, Phys. Rev. B 78, 020503 (2008).

${ }^{12}$ C. Tarantini, S. Lee, Y. Zhang, J. Jiang, C. W. Bark, J. D. Weiss, A. Polyanskii, C. T. Nelson, H. W. Jang, C. M. Folkman, S. H. Baek, X. Q. Pan, A. Gurevich, E. E. Hellstrom, C. B. Eom, and D. C. Larbalestier, Appl. Phys. Lett. 96, 142510 (2010).

${ }^{13}$ S. Lee, J. Jiang, J. D. Weiss, C. M. Folkman, C. W. Bark, C. Tarantini, A. Xu, D. Abraimov, A. Polyanskii, C. T. Nelson, Y. Zhang, S. H. Baek, H. W. Jang, A. Yamamoto, F. Kametani, X. Q. Pan, E. E. Hellstrom, A. Gurevich, C. B. Eom, and D. C. Larbalestier, Appl. Phys. Lett. 95, 212505 (2009).

${ }^{14}$ S. Lee, J. Jiang, Y. Zhang, C. W. Bark, J. D. Weiss, C. Tarantini, C. T. Nelson, H. W. Jang, C. M. Folkman, S. H. Baek, A. Polyanskii, D. Abraimov, A. Yamamoto, J. W. Park, X. Q. Pan, E. E. Hellstrom, D. C. Larbalestier, and C. B. Eom, Nature Mater. 9, 397 (2010).

${ }^{15}$ T. Matsui, H. Tanaka, N. Fujimura, T. Ito, H. Mabuchi, and K. Morii, Appl. Phys. Lett. 81, 2764 (2002).

${ }^{16}$ L. S. Darken and R. W. Gurry, J. Am. Chem. Soc. 67, 1398 (1945).

${ }^{17}$ Y. Tsujimoto, C. Tassel, N. Hayashi, T. Watanabe, H. Kageyama, K. Yoshimura, M. Takano, M. Ceretti, C. Ritter, and W. Paulus, Nature (London) 450, 1062 (2007)

${ }^{18}$ H. Kageyama, T. Watanabe, Y. Tsujimoto, A. Kitada, Y. Sumida, K. Kanamori, K. Yoshimura, N. Hayashi, S. Muranaka, M. Takano, M. Ceretti, W. Paulus, C. Ritter, and G. Andre, Angew. Chem., Int. Ed. 47, 5740 (2008).

${ }^{19}$ H. Zheng, Q. Zhan, F. Zavaliche, M. Sherburne, F. Straub, M. P. Cruz, L.-Q. Chen, U. Dahmen, and R. Ramesh, Nano Lett. 6, 1401 (2006). 